\title{
A phase plane analysis of the "Multi-bubbling" phenomenon in some slightly supercritical equations
}

\author{
Manuel del Pino \\ M. Del Pino - Departamento de Ingeniería Matemática and CMM, \\ Universidad de Chile, Casilla 170 Correo 3, Santiago, Chile. \\ E-mail: delpino@dim.uchile.cl \\ Jean Dolbeault \\ Ceremade (UMR CNRS no. 7534), Université Paris IX-Dauphine, \\ Place de Lattre de Tassigny, 75775 Paris Cédex 16, France \\ phone: (33) 1440546 78, fax: (33) 144054599 \\ E-mail: dolbeaul@ceremade.dauphine.fr \\ Monica Musso \\ Dipartimento di Matematica, Politecnico di Torino, \\ Corso Duca degli Abruzzi, 24 - 10129 Torino, Italy \\ E-mail: musso@calvino.polito.it
}

July 12, 2003

\begin{abstract}
The purpose of this paper is to present some recent results in two slightly supercritical problems known as the Brezis-Nirenberg problem in dimension $n \geq 3$ and an equation involving the exponential nonlinearity in dimension $n \geq 2$. For that purpose, we perform a phase plane analysis which emphasizes the common heuristic properties of the two problems, although more precise estimates can be obtained in some cases by variational methods.
\end{abstract}

Keywords. Brezis-Nirenberg problem, Gelfand problem, supercritical case, bifurcation diagram, singular solutions, Emden-Fowler transform, $p$-Laplacian, branches of solutions, critical and super-critical problems, dynamical systems, phase plane analysis, bubbles, spikes, multi-peaks, Lyapunov-Schmidt reduction

AMS classification (2000). Primary 35B40, 35B33, 35B25; Secondary: 35J60, 35B32, 35P 30 , $34 \mathrm{C} 23,35 \mathrm{~J} 20$

\section{Introduction}

The Brezis-Nirenberg problem

$$
\left\{\begin{aligned}
\Delta u+|u|^{p-1} u+\lambda u & =0 & & \text { in } B \\
u & =0 & & \text { on } \partial B
\end{aligned}\right.
$$

in the unit ball $B$ in $\mathbb{R}^{n}, n \geq 3$, is a well understood problem. Let

$$
p^{*}=\frac{n+2}{n-2}
$$


be the critical power and let $\lambda_{1}$ be the first eigenvalue of $-\Delta$ in $B$ with zero Dirichlet boundary conditions. Bounded positive solutions, which are all radial by [23], exist in the following cases, and only in these cases:

(i) Subcritical case: $p<p^{*}$ and $\lambda<\lambda_{1}$,

(ii) Critical case: $p=p^{*}$ and $\lambda \in\left(\lambda^{*}, \lambda_{1}\right)$, where $\lambda^{*}=0$ if $n \geq 4$ and $\lambda^{*}=\lambda_{1} / 4$ if $n=3$ : see $[5,2,1,9,10]$,

(iii) Supercritical case: $p>p^{*}$ and $\lambda \in\left(\lambda_{1}^{+}, \lambda_{1}\right)$, for some $\lambda_{1}^{+}>0$.

Radial solutions form a single branch bifurcating from $\lambda_{1} \in\left(0, \lambda_{1}\right)$, which is monotone decreasing if $p \leq p^{*}$ and with an asymptote at $\lambda=\lambda^{*}$, for some $\lambda^{*} \in\left[\lambda_{1}^{+}, \lambda_{1}\right)$ if $p \geq p^{*}$. Moreover, for $p>p^{*}$, there exists a radial singular positive solution if and only if $\lambda=\lambda^{*}$, see [27]. Note that other nonradial singular solutions are known to exists.

We are interested in describing the solutions in the slightly supercritical regime: $p=p^{*}+\varepsilon, \varepsilon \rightarrow 0$. The main phenomenon in this regime is the multi-bubbling phenomenon, which can be explained as follows. The equation

$$
\Delta U+U^{p^{*}}=0 \quad \text { in } \mathbb{R}^{n}
$$

has up to translations and dilations a unique positive solution which is radial:

$$
U(x)=\gamma_{n}\left(1+|x|^{2}\right)^{-(n-2) / 2} \quad \forall x \in \mathbb{R}^{n},
$$

where $\gamma_{n}=[n(n-2)]^{(n-2) / 4}$. In the limit $\varepsilon \rightarrow 0$, solutions of (1) turn out to be a superposition of such solutions. Namely, for any integer $k \geq 1$, solutions of the form

$$
u(x)=\sum_{j=1}^{k} M_{j} U\left(M_{j}^{2 /(n-2)} x\right)(1++o(1))
$$

can be found by variational methods for $\varepsilon>0$ and $\lambda>0$ small enough, if $n \geq 4$ [12]. The case $n=3$ is slightly different and has to be treated separately [14]. The numbers $M_{j}=M_{j}(\varepsilon)$ are such that

$$
\lim _{\varepsilon \rightarrow 0} M_{1}(\varepsilon)=+\infty \quad \text { and } \quad \lim _{\varepsilon \rightarrow 0} \frac{M_{j+1}(\varepsilon)}{M_{j}(\varepsilon)}=+\infty \quad \forall j=1,2, \ldots k-1 .
$$

Here $o(1)$ means that one can find a family of such solutions indexed by $\varepsilon$ such that the remainder term is uniformly small in $B$ as $\varepsilon \rightarrow 0$. Such a solution will be said to be a $k$-bubbles in the rest of this paper for reasons that will be made more clear after a convenient Emden-Fowler transform. The main feature of such a transformation is that it separates the scales. The first goal of this paper is to prove by ODE methods that all radial solutions behave like multi-bubbles in an appropriate limit.

Before going further, let us mention one more difference between the supercritical and the critical cases. For $p=p^{*}$, the bifurcation diagram is monotone decreasing in $L^{\infty}(B)$. For any $p=p^{*}+\varepsilon$, the same bifurcation diagram is not any more monotone decreasing but exhibits oscillations: see Fig. 1. The solutions that we are going to exhibit in the last section actually correspond to "turning points to the right" of the bifurcation diagram.

In dimension $n=2$, the nonlinearity which turns out to be critical is the exponential nonlinearity. The problem

$$
\left\{\begin{aligned}
\Delta u+\lambda e^{u} & =0 & & \text { in } B \\
u & =0 & & \text { on } \partial B
\end{aligned}\right.
$$


plays a role similar to (1) in the critical case $p=p^{*}(\varepsilon=0)$. Before going further, let us summarize the properties of the bifurcation diagram corresponding to (3). Since the pioneering work of Joseph and Lundgreen [25], it is well known that the solutions of (3) have different behaviours depending on the dimension: see [4] for a review of related results.

(i) If $n=2$, the branch of bounded solutions has an asymptote at $\lambda=0$ (see Fig. 2) and the equation has exactly two solutions for any $\lambda \in\left(0, \lambda_{1}^{+}\right)$. Solutions are explicit: see Section 3.

(ii) If $3 \leq n<10$, the branch of bounded solutions has an asymptote at $\lambda=\lambda^{*}>0$ $[9,10]$ (like in Fig. 2) and the equation has at least one solution for any $\lambda \in\left(0, \lambda_{1}^{+}\right)$, $\lambda_{1}^{+}>\lambda^{*}$. This branch oscillates around $\lambda=\lambda^{*}$.

(iii) If $n \geq 10$, the branch of bounded solutions has an asymptote at $\lambda=\lambda^{*}>0$ and the equation has exactly one solution for any $\lambda \in\left(0, \lambda^{*}\right)$.

All these properties will be easy consequences of the phase plane analysis of Section 3. Moreover, it is also known that for $n>2$, there exists a unique $\lambda=2(n-2)$ for which there exists a radial singular solution $u^{*}$. Of course $\lambda=\lambda^{*}=2(n-2)$ is the asymptote of the branch of bounded radial solutions. This solution is moreover explicit (for $n>2$ ) and such that

$$
e^{u^{*}(x)}=\frac{1}{|x|^{2}}
$$

see [28]. Since we are dealing with radial solutions, Equation (3) is strictly equivalent to

$$
\left\{\begin{array}{l}
u^{\prime \prime}+\frac{n-1}{r} u^{\prime}+\lambda e^{u}=0, \quad r \in(0,1) \\
u^{\prime}(0)=0, \quad u(1)=0
\end{array}\right.
$$

and it is very natural to consider now $n$ as a real parameter. If we look at the bifurcation diagram, then $n=2$ appears as the critical case, while $n=2+\varepsilon, \varepsilon>0$ is supercritical. Note that looking for radial solutions of the equation

$$
|x|^{N-2-\varepsilon} \operatorname{div}\left(|x|^{-(N-2-\varepsilon)} \nabla u\right)+\lambda e^{u}=0
$$

in the unit ball of $\mathbb{R}^{N}, N \in \mathbb{N}$, with zero Dirichlet boundary conditions, is exactly equivalent to (4) with $n=2+\varepsilon$. However, we shall rather consider radial solutions corresponding to the more general supercritical equation

$$
\left\{\begin{array}{c}
\Delta_{p} u+\lambda e^{u}=0 \quad \text { in } B \\
u>0, \quad u=0 \quad \text { on } \partial B
\end{array}\right.
$$

with $p<p^{*}:=n$, written in radial coordinates. Here we use the standard notation $\Delta_{p} u:=\operatorname{div}\left(|\nabla u|^{p-2} \nabla u\right)$ : see Equation (12) for the corresponding ODE problem. In the rest of this paper, we shall assume that $p>1$. Both $p$ and $n$ will be considered as real parameters and the small parameter in the slightly supercritical regime is now $\varepsilon=p^{*}-p=n-p>0$. We are interested in understanding how the supercritical regime approaches the critical regime, i.e., in what happens when $n$ approaches 2 from above for $p=2$ fixed, or what happens when $p \rightarrow p^{*}=n$ from below, $n>1$ fixed. Equations (1) and (5) turn out to have very similar bifurcation diagrams and both exhibit a multi-bubbling phenomenon in the slightly supercritical regime, in the sense that there exist $k$ functions $v_{j}, j=1,2, \ldots k$ and a sequence of $k$ parameters $\mu_{j}(\varepsilon)$, $j=1,2, \ldots k$, for which

$$
\lim _{\varepsilon \rightarrow 0}\left[\mu_{j+1}(\varepsilon)-\mu_{j}(\varepsilon)\right]=+\infty \quad \forall j=1,2, \ldots k-1
$$


such that there exists a solution $u$ of (5) which takes the form

$$
e^{u}(x)=\frac{1}{|x|^{p}}\left[\sum_{j=1}^{k} v_{j}\left(\log r+\mu_{j}(\varepsilon)\right)\right](1+o(1)) .
$$

Actually the result holds true for any solution of (5) and any appropriate $\lambda$, except that the convergence in $\varepsilon$ only holds for sequences $\left(\varepsilon_{n}\right)_{n \in \mathbb{N}}$ with $\lim _{n \rightarrow \infty} \varepsilon_{n}=0$. Moreover, it is an open question to check if the above $k$-bubbles asymptotic profile depends on the sequence or not - in other words if the function $v_{j}$ are uniquely defined for a given $j$ or not. However, and this is the main difference with the Brezis-Nirenberg problem, it turns out that these functions differ from each other and for instance the sequence $\left(\max v_{j}\right)_{j}$ is strictly decreasing.

The approach we will take in this paper is to consider the two problems as pure phase plane analysis questions. This makes the analogy between the Brezis-Nirenberg problem and the case of the exponential nonlinearity easier to understand: the bubbles appear as natural objects. However, getting precise estimates turns out to be difficult, so that asymptotic energy expansions and Lyapunov-Schmidt reductions are actually more accurate, at least in the case of the Brezis-Nirenberg problem. Such an approach is still to be done in the exponential case. To avoid technicalities, we will not give all details of the proofs.

In the context of slightly supercritical problems, multi-bubbling has already been observed in previous works $[12,14,15,16]$ on the Brezis-Nirenberg problem using a Lyapunov-Schmidt reduction. It is also expected to occur in non-radial frameworks, with eventually weights, and this is the subject of an ongoing study [13] using also a Lyapunov-Schmidt reduction. Here, since the problem is reduced to an ODE, we use more direct methods based on phase plane analysis. By the mean of Emden-Fowler changes of variables, it is indeed possible to reduce the study of radial solutions of (1) and (5) to the study of ODE systems whose qualitative behaviour is completely understood. Moreover, these ODE systems are autonomous in the case of the exponential nonlinearity, which allows to describe in a very precise way how the supercritical regime $p<p^{*}=n$ approaches the critical case $p=p^{*}$. The two main features of the exponential nonlinearity compared to power laws is that the phase plane analysis is almost explicit and that the bubbles do not present the same shape. Further references will be given throughout the rest of the text.

This paper is organized as follows. Using the Emden-Fowler change of variables, we first show in Section 2 how bubbles appear and why they all have the same height in the case of the Brezis-Nirenberg problem. Then we will consider the case of the exponential nonlinearity in Section 3 (but we are not going to prove here that bubbles have different heights). In the last section, we state some results based on variational approaches in the case of the Brezis-Nirenberg problem, which are reproduced from [12], and finally formulate an open problem for the exponential nonlinearity.

\section{Phase plane analysis: the Brezis-Nirenberg problem}

This section is intended to give an analysis of the multi-bubbling phenomenon in the case of the Brezis-Nirenberg problem by ODE methods. Throughout this section, $p^{*}=\frac{n+2}{n-2}$ is the critical exponent and $n \geq 3$ is an integer.

\subsection{The Emden-Fowler change of variables}

Consider the bounded radial solutions of the Brezis-Nirenberg problem in the unit ball $B \subset \mathbb{R}^{n}, n \geq 3$, with zero Dirichlet boundary conditions. In terms of $r=|x|, x \in \mathbb{R}^{n}$, 
the solutions are given by

$$
\left\{\begin{array}{l}
u^{\prime \prime}+\frac{n-1}{r} u^{\prime}+|u|^{p-1} u+\lambda u=0, \quad r \in(0,1) \\
u^{\prime}(0)=0, \quad u(1)=0
\end{array}\right.
$$

The Emden-Fowler transformation $r=e^{s}, u(r)=r^{-2 /(p-1)} v(s)$ changes the problem into

$$
\left\{\begin{array}{l}
v^{\prime \prime}+|v|^{p-1} v-\beta v=-\alpha v^{\prime}-\lambda e^{2 s} v, \quad s \in(-\infty, 0) \\
v(0)=0, \quad \lim _{s \rightarrow-\infty} e^{-2 s /(p-1) s} v(s)=u(0):=\mu>0, \\
\lim _{s \rightarrow-\infty} e^{-s(p+1) /(p-1)}\left(v^{\prime}(s)-\frac{2}{p-1} v(s)\right)=0
\end{array}\right.
$$

where $\alpha=n-2-4 /(p-1)$ and $\beta=2[(n-2) p-n] /(p-1)^{2}$ are positive whenever $p>(n+2) /(n-2)$ and $p>n /(n-2)$ respectively. Let

$$
p=p^{*}+\varepsilon=\frac{n+2}{n-2}+\varepsilon
$$

and consider the slightly supercritical case $\varepsilon>0$, small. With evident notations,

$$
\alpha(\varepsilon)=\frac{(n-2)^{2}}{4+\varepsilon(n-2)} \varepsilon \quad \text { and } \quad \beta(\varepsilon)=2 \frac{(n-2)^{2}(2+\varepsilon(n-2))}{(4+\varepsilon(n-2))^{2}} .
$$

We are going to describe the behaviour of the solutions in the critical limit $\varepsilon \rightarrow 0$ using a phase plane analysis. This description is quite standard, see for instance [8, 22, 25] and references therein. The novelty in our approach is to use it to handle the critical limit $\varepsilon \rightarrow 0$.

Let $x(s)=v(s), y(s)=v^{\prime}(s)$, where $v$ is a solution of $(7)$ :

$$
\left\{\begin{array}{l}
x^{\prime}=y \\
y^{\prime}=-\left(|x|^{p-1} x-\beta x\right)-\lambda e^{2 s} x-\alpha y
\end{array}\right.
$$

supplemented with boundary conditions which are now translated into

$$
\left\{\begin{array}{l}
x(0)=0, \quad \lim _{s \rightarrow-\infty} e^{-2 s /(p-1)} x(s)=\mu>0 \\
\lim _{s \rightarrow-\infty} e^{-s(p+1) /(p-1)}\left[y(s)-\frac{2}{p-1} x(s)\right]=0
\end{array}\right.
$$

\section{Remark}

(i) Note that if $u$ is a bounded solution of (6), $\lim _{s \rightarrow-\infty} y(s) / x(s)=2 /(p-1)$ does not depend on $\lambda$.

(ii) Each time the curve $s \mapsto(x(s), y(s))=: z(s)$ crosses the line $y=0$ (resp. $x=0$ ) at some point such that $x \neq 0($ resp. $y \neq 0)$, then $x^{\prime}=0$ (resp. $y^{\prime}=0$ if $\left.p=p^{*}\right)$ so that $z$ has an horizontal (resp. vertical) tangent line.

(iii) The condition $x(0)=0$ determines the value of the shooting parameter $\mu=\mu(\lambda)$ for any given $\lambda$.

Let us temporarily write $z=z_{\lambda, \mu}$ to emphasize the dependence of $z$ in the parameters $\lambda$ and $\mu$, which show up in (8) and (9). The condition $x(0)$ turns out to be not very easy to handle, since the parameter $\mu=\mu(\lambda)$ has to be adjusted accordingly. To parametrize the solutions, it is more convenient to proceed as follows. Consider 
(8)-(9) with $\lambda=1$ and take $\mu>0$ as a shooting parameter at $s=-\infty$. Define $s_{0}(\mu):=\inf \{s \in \mathbb{R}: x(s)=0\}$. If $s_{0}(\mu)$ is finite, then

$$
z_{\lambda, \mu(\lambda)}(s)=z_{1, \mu}\left(s-s_{0}(\mu)\right) \quad \text { if and only if } \quad \lambda=e^{2 s_{0}(\mu)} .
$$

The above invariance is actually a well known fac. Namely the branch of the solutions of (6) can similarly be parametrized as follows [30,3]. Let $u_{a}$ be the solution in $\mathbb{R}^{+}$of

$$
\left\{\begin{array}{l}
u_{a}^{\prime \prime}+\frac{n-1}{r} u_{a}^{\prime}+\left|u_{a}\right|^{p-1} u_{a}+u_{a}=0, \quad r \in \mathbb{R}^{+} \\
u_{a}^{\prime}(0)=0, \quad u_{a}(0)=a>0
\end{array}\right.
$$

and consider its first zero $\rho=\rho(a): u_{a}(\rho)=0, u_{a}(r)>0$ for any $r \in(0, \rho)$. Then

$$
u(r)=\rho^{-2 /(p-1)} u_{a}(r \rho)
$$

is the unique solution of $(6)$ with $u(0)=a \rho^{-2 /(p-1)}$ and $\lambda=\rho^{2}$. Note that the uniqueness $[31,26,32]$ of the solutions of (1) in turn means that $a \mapsto \rho(a)$ is monotone decreasing.

\subsection{The critical case}

From [5], it is known that positive bounded solutions of (1) with $p=p^{*}, \varepsilon=0$, exist for any $\lambda \in\left(\lambda^{*}, \lambda_{1}\right)$, where $\lambda_{1}$ is the first eigenvalue of $-\Delta$ in the unit ball of $\mathbb{R}^{n}$ with zero Dirichlet boundary conditions, and $\lambda^{*}=0$ if $n \geq 4, \lambda^{*}=\lambda_{1} / 4$ if $n=3$. The study done in [3] (also see [18]) shows that for any given $\lambda \in\left(\lambda^{*}, \lambda_{1}\right)$, solutions of (8) with $x(0)=0$ and $y(0)=-\gamma<0$ exhibit only three possible behaviours. In terms of the original coordinates, these solutions are given by

$$
\left\{\begin{array}{l}
u^{\prime \prime}+\frac{n-1}{r} u^{\prime}+|u|^{p-1} u+\lambda u=0, \quad r \in(0,1) \\
u(1)=0, \quad u^{\prime}(1)=-\gamma
\end{array}\right.
$$

Define the energy by

$$
E(s)=\frac{1}{2}|y(s)|^{2}+\frac{1}{p+1}|x(s)|^{p+1}-\frac{\beta}{2}|x(s)|^{2}
$$

and consider its limiting value

$$
\mathcal{E}_{\gamma, \lambda}=\lim _{s \rightarrow-\infty} E(s)
$$

If $\lambda \in\left(\lambda^{*}, \lambda_{1}\right)$ is fixed, then there exists a positive $\gamma_{*}(\lambda)$ such that

(i) If $\gamma \in\left(0, \gamma_{*}(\lambda)\right)$, then $\mathcal{E}_{\gamma, \lambda}<0$ and the solution of (10) is a positive solution of (6), which is singular at the origin.

(ii) If $\gamma \in\left(\gamma_{*}(\lambda),+\infty\right)$, then $\mathcal{E}_{\gamma, \lambda}>0$ and the solution of (10) is a sign-changing solution of (6), which is singular and oscillating at the origin (it changes sign infinitely many times).

(iii) If $\gamma=\gamma_{*}(\lambda)$, then $\mathcal{E}_{\gamma, \lambda}=0$ and the solution of (10) is the unique positive bounded solution of (6).

\subsection{The slightly supercritical case}

Let $\lambda \in\left(\lambda^{*}, \lambda_{1}\right)$ and consider $\gamma<\gamma_{*}(\lambda)$ so that $\mathcal{E}_{\gamma, \lambda}<0$. Consider $T>0$ fixed such that on $(-T, 0)$ the solution $z_{0}=\left(x_{0}, y_{0}\right)$ of $(8)$ in the critical case $\left(p=p^{*}, \varepsilon=0\right)$ is such that $y_{0}$ changes sign $k_{T}$ times. Take $\varepsilon>0$, small enough and consider the 
solution of (8) for $p=p^{*}+\varepsilon$, with $\varepsilon$ small enough so that $y$ also changes $k_{T}$ times on $(-T, 0)$. The number $k_{T}$ can be taken arbitrarily large for $T$ large enough, since $z_{0}$ is asymptotically periodic as $s \rightarrow-\infty$.

According to Merle and Peletier [27], for any $\varepsilon>0$ given, there exists a unique $\lambda^{*}(\varepsilon)$ such that (6) has a positive singular radial solution. Moreover, $\lambda^{*}(\varepsilon)$ is the asymptote of the bifurcation curve corresponding to large $L^{\infty}$ norms (consistently, we shall write $\lambda^{*}=\lambda^{*}(0)$ in the critical case). Therefore, if $\lambda \neq \lambda^{*}(\varepsilon)$, either the solution of (6) corresponding to $u$ is bounded or it has to change sign and the curve $s \mapsto z(s)$ crosses the axis $x=0$ for some $y>0$. Let $k$ be the number of sign changes of $y$ before $x$ becomes negative. It is clear that $k$ can be taken arbitrarily large and, as a consequence, larger than $k_{T}$.

For $\varepsilon>0$, by increasing $\gamma$, we may find a solution such that $x$ is positive for any $s \in \mathbb{R}^{-}$and $y$ changes sign at least $k$ times. Such a solution is a multi-bubbles solution in the limit $\varepsilon \rightarrow 0$.

Theorem 1 Assume that $n \geq 3, p^{*}=\frac{n+2}{n-2}$. With the above notations, for any $\varepsilon>0$ small enough, for any integer $\kappa \geq 1$, if $\lambda^{n-2}\left(\lambda^{*}, \lambda_{1}\right)$ and $\lambda \neq \lambda^{*}(\varepsilon)$, then there exists a solution $z=(x, y)$ of (8) with $p=p^{*}+\varepsilon$, which can be written as

$$
x(s)=x_{0}^{\lambda}(s)+\sum_{i=1}^{k} x^{*}\left(s+s_{i}(\varepsilon)\right)+o(1)
$$

for some $k \geq \kappa$, where $x_{0}^{\lambda}=x_{0}$ is the bounded solution corresponding to (8) in the critical case $\left(p=p^{*}, \varepsilon=0\right)$ with $\gamma=\gamma^{*}(\lambda)$ and $x^{*}$ is the unique positive solution of the asymptotic problem

$$
x^{\prime \prime}+|x|^{p^{*}-1} x-\frac{1}{4}(n-2)^{2} x=0
$$

such that $x^{\prime}(0)=0$ and with zero energy: $\frac{1}{2}\left|x^{\prime}\right|^{2}+\frac{n-2}{2 n}|x|^{2 n /(n-2)}-\frac{1}{8}(n-2)^{2}|x|^{2}=0$. By o(1), we mean that the convergence holds uniformly on $(-\infty, 0) \ni s$. This implies that the difference of the corresponding solution $u$ of (6) and of its asymptotic form, namely

$$
r^{-2 /(p-1)}\left[x_{0}^{\lambda}(\log r)+\sum_{i=1}^{k} x^{*}\left(\log r+s_{i}(\varepsilon)\right)\right]
$$

also uniformly converges to 0 in the unit ball.

Moreover, in dimension $n \geq 4$, if we consider the case where $\lambda=\lambda(\varepsilon)>0$ is itself a function of $\varepsilon$ such that $\lim _{\varepsilon \rightarrow 0} \lambda(\varepsilon)=0$, then for $p=p^{*}+\varepsilon$ the solution $x_{0}^{\lambda(\varepsilon)}$ of

$$
\left\{\begin{array}{l}
x^{\prime \prime}+|x|^{p-1} x-\beta(\varepsilon) x+\lambda(\varepsilon) e^{2 s} x=0 \\
x(0)=0, \quad x^{\prime}(0)=-\gamma^{*}(\lambda(\varepsilon))
\end{array}\right.
$$

uniformly converges to $x^{*}$ in $\mathbb{R}^{-}$.

Proof. We only give the main steps at a heuristic level since detailed computations turn out to be very tedious. The solutions that we consider correspond to $\varepsilon>0$ unless it is specified, and explicitly depend on $\varepsilon$. The fact that $k$ can be chosen arbitrarily large follows from the estimate for $k_{T}$ (see [3] for more details). Then the curve $z$ necessarily converges to the zero energy curve for the asymptotic problem, except for the first bubble, which allows to characterize $x^{*}$. The proof of these facts goes as follows.

First of all, take $\varepsilon>0$ small enough in order that for $T>0$ given, large enough, the solution is such that $y$ changes $\operatorname{sign} \kappa=k_{T}$ times on $(-T, 0)$ (note again that $\kappa$ can be chosen arbitrarily large by taking $T$ large enough). Actually, $y$ changes sign $k$ times 
for some $k \geq \kappa$ before $x$ becomes negative. Increase now $\gamma=-y(0)$. From [3] and after eventually decreasing further $\varepsilon>0$, it is clear that there is an upper bound on $\gamma$ for which the above properties hold true. This simply means that there is a point of the curve $z$ corresponding to some $s=s(\varepsilon) \rightarrow-\infty$ as $\varepsilon \rightarrow 0$, which converges to $(0,0)$ : $\lim _{\varepsilon \rightarrow 0} z(s(\varepsilon))=(0,0)$. What one has to prove is that there are at least $k$ different such points, which all converge to $(0,0)$ as $\varepsilon \rightarrow 0$.

By energy methods as in [3], it is immediate that for $\tilde{s}=\tilde{s}(\varepsilon)$ such that $x(\tilde{s}(\varepsilon)) \geq 0$ and $y(\tilde{s}(\varepsilon))=0$, then $\lim _{\varepsilon \rightarrow 0} x(\tilde{s}(\varepsilon))=0$ if $\tilde{s}(\varepsilon) \geq s(\varepsilon)$. This also holds true for $\tilde{s}(\varepsilon)<s(\varepsilon)$ as can be shown by contradiction, by taking the limit case $\varepsilon \rightarrow 0$.

The last statement on the convergence of $x_{0}^{\lambda(\varepsilon)}$ to $x^{*}$ is easy.

As an easy corollary of the above proof, we can state the following

Corollary 2 Assume that $n \geq 3$. With the above notations, the range in $\lambda$ for which multi-bubble solutions of (1) with an arbitrarily large number of bubbles exist is actually $\left(\lambda^{*}, \lambda_{1}\right)$ in the limit $\varepsilon \rightarrow 0, \varepsilon>0$.

Proof. It is an easy consequence of (11). Details are left to the reader. Note that the solutions take a slightly more general form than in (2), since the first bubble for a $\lambda>\lambda^{*}$ corresponds to a positive solution of the critical case $p=p^{*}$, for the same value of $\lambda$.

In other words, the bifurcation diagram in $L^{\infty}(B)$ is asymptotically oscillating within its maximal values, in the limit $\varepsilon \rightarrow 0, \varepsilon>0$ and for large $L^{\infty}(B)$-norms of the solutions.

\section{Remark}

(i) The function $x^{*}$ is explicit:

$$
x^{*}(s)=\gamma_{n}\left(e^{s}+e^{-s}\right)^{-(n-2) / 2} \quad \forall s \in \mathbb{R},
$$

where $\gamma_{n}=(n(n-2))^{(n-2) / 4}$. The uniqueness is a consequence of the fact that $x^{*}(0)=(n(n-2) / 4)^{(n-2) / 4}$ and $x^{* \prime}(0)=0$, which is itself a consequence of the boundary conditions and the fact that $x^{*}$ has zero energy.

(ii) In dimension $n=3, x_{0}^{\lambda(\varepsilon)}$ converges to some function $x_{0}^{*} \neq x^{*}$, which is also explicit [5, 3]. This case is very special and $x_{0}^{*}$ has to do with Robin's function [19].

(iii) For $\lambda=\lambda(\varepsilon) \searrow 0$, a much more precise statement can actually be given using a Lyapunov-Schmidt reduction: see [12, 14] and Section 4 of this paper.

The fact that there are $k$-bubbles for any $k \geq 1$ in the limit $\varepsilon \rightarrow 0, \varepsilon>0$ is a delicate issue at the level of the phase space analysis, since it involves a good understanding of the mechanism responsible for the change of the number of nodes in the singular limit $\varepsilon \rightarrow 0$. On the contrary, such a result will be one of the main features of the variational methods, see Section 4 .

The assumption $\lambda \neq \lambda(\varepsilon)$ is clearly technical and should be removable to the price of some further analysis.

An interesting feature of the Brezis-Nirenberg problem is that all bubbles except the first one are the same, up to translations. This is somehow standard in such multi-scales concentration problems. As we will see in the next section, the case of the exponential nonlinearity differs on that point. See [13] for further details. 


\section{Phase plane analysis: the exponential nonlinearity}

This section is devoted to the equation

$$
\Delta_{p} u+\lambda e^{u}=0
$$

in $H_{0}^{1}(B)$, in the slightly supercritical case $\varepsilon=p^{*}-p>0,1<p<p^{*}$, where the critical parameter is now

$$
p^{*}=n
$$

\subsection{The generalized Emden-Fowler change of variables}

Since (5) is invariant under rotations, for bounded solutions it is possible to restrict the study to the case of radial solutions. See [11] and [7] for some recent result on symmetry properties of the solutions. Let $u$ be a solution of

$$
\left\{\begin{array}{l}
\Delta_{p, n} u+\lambda e^{u}=0, \quad r \in(0,1) \\
u(0)>0, \quad \frac{d u}{d r}(0)=0, \quad u(1)=0
\end{array}\right.
$$

where

$$
\Delta_{p, n} u:=\frac{1}{r^{n-1}} \frac{d}{d r}\left(r^{n-1}\left|\frac{d u}{d r}\right|^{p-2} \frac{d u}{d r}\right)=\left|\frac{d u}{d r}\right|^{p-2}\left((p-1) \frac{d^{2} u}{d r^{2}}+\frac{n-1}{r} \frac{d u}{d r}\right)
$$

(at least if $\frac{d u}{d r} \neq 0$ ) is the radial version of $\Delta_{p}$ in dimension $n$. Here we abusively use the same notations for the solutions of (5) and (12). As long as we consider solutions of (12), it is possible to see $p$ and $n$ as two independent real parameters. Let $r=e^{s}$, $s \in(-\infty, 0]$ and define $v(s):=u(r)$. Then (12) is equivalent to

$$
\left\{\begin{array}{l}
(p-1)\left|v^{\prime}\right|^{p-2} v^{\prime \prime}+(n-p)\left|v^{\prime}\right|^{p-2} v^{\prime}+\lambda e^{v+p s}=0, \quad s \in(-\infty, 0) \\
\lim _{s \rightarrow-\infty} v(s)>0, \quad \lim _{s \rightarrow-\infty} e^{-s} v^{\prime}(s)=0, \quad v(0)=0
\end{array}\right.
$$

where $v^{\prime}=\frac{d v}{d s}$. Note that the change of variables means that

$$
\lim _{s \rightarrow-\infty} v(s)=u(0) .
$$

The equation for $v$ can be reduced to an autonomous ODE system as follows. Let

$$
x(s)=\lambda e^{v(s)+p s} \quad \text { and } \quad y(s)=\left|v^{\prime}(s)\right|^{p-2} v^{\prime}(s) .
$$

Then

$$
\left\{\begin{array}{l}
x^{\prime}=x\left(v^{\prime}+p\right) \\
y^{\prime}=(p-1)\left|v^{\prime}\right|^{p-2} v^{\prime \prime}
\end{array}\right.
$$

and (12) is finally equivalent to the system

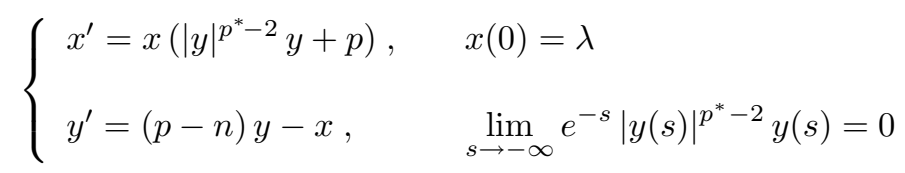

where $p^{*}=\left(1-\frac{1}{p}\right)^{-1}$ is the Hölder conjugate of $p$, so that

$$
y=\left|v^{\prime}\right|^{p-2} v^{\prime} \Longleftrightarrow v^{\prime}=|y|^{p^{*}-2} y .
$$

As in the case of the Brezis-Nirenberg problem, the change of coordinates is somewhat classical, see for instance $[8,25]$, at least for $p=2$. The novelty of our approach is to use it in order to understand the limit $n-p=\varepsilon \rightarrow 0, \varepsilon>0$. 


\subsection{Parametrization of the solutions}

The behaviour of the solutions easily follows from the study of the vector field and a linearization around the two fixed points: $P^{-}=(0,0)$ and $P^{+}=p^{p-1}(n-p,-1)$. The linearization of $(13)$ at $P^{-}$is

$$
\left(\begin{array}{l}
X \\
Y
\end{array}\right)^{\prime}=\left(\begin{array}{cc}
p & 0 \\
-1 & -(n-p)
\end{array}\right)\left(\begin{array}{l}
X \\
Y
\end{array}\right)
$$

with eigenvalues $p$ and $-(n-p)$, and, at $P^{+}$,

$$
\left(\begin{array}{l}
X \\
Y
\end{array}\right)^{\prime}=\left(\begin{array}{cc}
0 & p(n-p) /(p-1) \\
-1 & -(n-p)
\end{array}\right)\left(\begin{array}{l}
X \\
Y
\end{array}\right)
$$

with eigenvalues

$$
\frac{1}{2} \sqrt{n-p}(-\sqrt{n-p} \pm i \sqrt{p(p+3) /(p-1)-n})
$$

This proves the

Lemma 3 Assume that $p<n<p(p+3) /(p-1)$. Then the following properties hold: (i) $P^{-}$is hyperbolic and $P^{+}$is elliptic.

(ii) Any trajectory of (13) is such that $x(s)$ does not change sign. Any trajectory with $x>0$ enters the lower quadrant corresponding to $x>0, y<0$.

(iii) $P^{+}$(resp. $P^{-}$) is attracting all trajectories with $x>0$ as $s \rightarrow+\infty$ (resp. all bounded trajectories with $x>0$ as $s \rightarrow-\infty)$.

(iv) There exists a bounded trajectory $s \mapsto(x(s), y(s))$ such that

$$
\lim _{s \rightarrow \pm \infty}(x(s), y(s))=P^{ \pm}
$$

This heterocline trajectory is unique, up to any translation in s.

Note that for $n>p(p+3) /(p-1)$, to the linearization of (13) at $P^{+}$correspond two negative eigenvalues, so that the trajectory connecting $P^{-}$to $P^{+}$is unique, up to any translation in $s$, and monotone in $y$. As a consequence, we recover for instance that for $p=2, n \geq 10$, the branch of the solutions of $(5)$ in $L^{\infty}(\Omega)$ is monotone. From now on we assume that $p \leq n<p(p+3) /(p-1)$. Let $(\bar{x}, \bar{y})$ be the unique trajectory such that $\lim _{s \rightarrow-\infty}(\bar{x}(s), \bar{y}(s))=P^{-}$and $\bar{x}(0)=\max _{s \in \mathbb{R}} \bar{x}(s)$. In order to emphasize the dependence in $\varepsilon$, we shall write $\left(\bar{x}^{\varepsilon}, \bar{y}^{\varepsilon}\right)$ whenever needed.

Lemma 4 Assume that $p \leq n<p(p+3) /(p-1)$. For a given $\lambda$, to any solution $v$ of (12) corresponds a unique $s_{0}$ such that

$$
\lambda e^{v(s)+p s}=\bar{x}\left(s+s_{0}\right)
$$

for any $s \leq 0$. Reciprocally, for any $\lambda \in\left(0, \lambda_{1}^{+}\right]$, where $\lambda_{1}^{+}:=\max _{s \in \mathbb{R}} \bar{x}(s)=\bar{x}(0)$, the equation $\bar{x}\left(s_{0}\right)=\lambda$ has at least one solution and

$$
v(s)=\log \left(\frac{1}{\lambda} \bar{x}\left(s+s_{0}\right)\right)-p s
$$

is a solution of (12).

Note that with the change of variables $s=t-s_{0}$,

$$
v\left(t-s_{0}\right)=\log \left(\frac{\bar{x}(t)}{\bar{x}\left(s_{0}\right)}\right)-p t+p s_{0} \quad \forall t \in\left(-\infty, s_{0}\right) .
$$


The corresponding solution $u$ of (5) is fully determined by $\lambda=\bar{x}\left(s_{0}\right), u^{\prime}(0)=0$ and

$$
u(0)=\lim _{t \rightarrow-\infty} v\left(t-s_{0}\right)=\lim _{t \rightarrow-\infty} \log \left(\frac{\bar{x}(t) e^{-p t}}{\bar{x}\left(s_{0}\right) e^{-p s_{0}}}\right) .
$$

The parametrization in Lemma 4 is a straightforward consequence of the EmdenFowler change of coordinates. The next result only involves an elementary phase plane analysis.

Lemma 5 Let $\lambda^{*}=p^{p-1}(n-p)$. Assume that $p<n<p(p+3) /(p-1)$. There exists two sequences $\left(\lambda_{k}^{-}\right)_{k \geq 1}$ and $\left(\lambda_{k}^{+}\right)_{k \geq 1}$ such that:

(i) $\left(\lambda_{k}^{-}\right)_{k \geq 1}$ is increasing and $\lim _{k \rightarrow+\infty} \lambda_{k}^{-}=\lambda^{*}$.

(ii) $\left(\lambda_{k}^{+}\right)_{k \geq 1}$ is decreasing and $\lim _{k \rightarrow+\infty} \lambda_{k}^{+}=\lambda^{*}$.

(iii) Equation (12) has no solutions if $\lambda>\lambda_{1}^{+}, 2 k-1$ solutions if $\lambda=\lambda_{k}^{+}$or $\lambda \in$ $\left(\lambda_{k-1}^{-}, \lambda_{k}^{-}\right)$with the convention $\lambda_{0}^{-}=0$, and $2 k$ solutions if $\lambda=\lambda_{k}^{-}$or $\lambda \in\left(\lambda_{k+1}^{+}, \lambda_{k}^{+}\right)$, $k \geq 1$.

(iv) Equation (12) has infinitely many solutions if and only if $\lambda=\lambda^{*}$.

Assume next that $p=n$ and let $\lambda_{1}^{+}:=\sup _{s \in \mathbb{R}} \bar{x}(s)$. Then Equation (12) has no solutions if $\lambda>\lambda_{1}^{+}$, one and only one solution if $\lambda=\lambda_{1}^{+}$and two and only two solutions if $\lambda \in\left(0, \lambda_{1}^{+}\right)$.

The supercritical case (see Figs. 1 and 2) exhibits a behaviour similar to the one of the supercritical Brezis-Nirenberg problem, while in the critical case $p=n,(13)$ becomes an Hamiltonian system:

$$
x^{\prime}=x\left(|y|^{p^{*}-2} y+p\right), \quad y^{\prime}=-x
$$

which is explicitely solvable in the case $p=2: u(r)=2 \log \left(\frac{a^{2}+1}{a^{2}+r^{2}}\right)$ is a solution of (5) for any $a>0$ such that $\lambda=\frac{8 a^{2}}{\left(a^{2}+1\right)^{2}}$.

\subsection{Description of the critical limit}

This regime corresponds to the limit $\varepsilon=n-p \rightarrow 0, \varepsilon>0$ and it is clearly the delicate part of our ODE approach. We shall only expose the crucial steps and refer to [13] for complete proofs.

For any $\epsilon>0$, define by $s_{k}(\varepsilon)$ the sequence of the points of local maxima of $\bar{x}^{\varepsilon}$. By definition of $\left(\bar{x}^{\varepsilon}, \bar{y}^{\varepsilon}\right), s_{1}(\varepsilon)=0$ for any $\varepsilon>0$. It is then easy to check that

Lemma 6 For any $k \geq 1$,

$$
\lim _{\varepsilon \rightarrow 0}\left[s_{k+1}(\varepsilon)-s_{k}(\varepsilon)\right]=+\infty .
$$

To study the critical limit, we emphasize the dependence in $\varepsilon$. Let $\lambda_{k}^{\varepsilon,+}=\bar{x}^{\varepsilon}\left(s_{k}(\varepsilon)\right)$. According to Lemma $4,\left(\lambda_{k}^{\varepsilon,+}\right)_{k \geq 1}$ is a positive decreasing sequence. Define $\bar{\lambda}_{k}^{+}:=$ $\lim _{\varepsilon \rightarrow 0} \lambda_{k}^{\varepsilon,+}$.

It is actually not clear that for any sequence $\left(\varepsilon_{n}\right)_{n \in \mathbb{N}}$ with $\varepsilon_{n}>0, \lim _{n \rightarrow \infty} \varepsilon_{n}=0$, the limit of $\lambda_{k}^{\varepsilon_{n},+}$ is unique and well defined so that one should consider a special sequence $\left(\varepsilon_{n}\right)_{n \in \mathbb{N}}$ and eventually extract subsequences. For the sake of simplicity, we will speak of "the limit $\varepsilon \rightarrow 0$ " in the rest of this section, except in the statement of Theorem 10.

Lemma 7 For any $k \geq 1, \bar{\lambda}_{k}^{+}>0$ and $\bar{\lambda}_{1}^{+}=\lambda_{1}^{0,+}$. Moreover $\left(\bar{\lambda}_{k}^{+}\right)_{k \in \mathbb{N}}$ is a strictly decreasing sequence.

Proof. The general strategy is the following. Prove first that the energy decays at most at an exponential rate proportional to $\varepsilon$ and prove that the angular velocity with respect to $P^{+}$is bounded from below by a constant which is also proportional to $\varepsilon$. 
This is actually not true a priori and a much more detailed analysis is required for any $k \geq 2$. This proves that $\bar{\lambda}_{k}^{+}$is positive for any $k \geq 1$ : we shall say that multi-bubbling occurs. Arguing by contradiction, one can then show that the energy has to decay so that the sequence $\left(\bar{\lambda}_{k}^{+}\right)_{k>1}$ is strictly decreasing: we shall say that bubbles dont have the same "height". We refer to [13] for further details, which are quite lengthy.

Corollary 8 For any $k \geq 1$, as $\varepsilon \rightarrow 0$,

$$
\bar{x}^{\varepsilon}(s) \rightarrow \sum_{j=1}^{k} x_{j}\left(s-s_{j}(\varepsilon)\right)
$$

uniformly on any interval $(-\infty, a(\varepsilon)) \in \mathbb{R}$ such that $s_{k}(\varepsilon)<a(\varepsilon)<s_{k+1}(\varepsilon)$ with $\liminf _{\varepsilon \rightarrow 0}\left(s_{k+1}(\varepsilon)-a(\varepsilon)\right)=\liminf _{\varepsilon \rightarrow 0}\left(a(\varepsilon)-s_{k}(\varepsilon)\right)=+\infty$.

Let $s_{0, k}^{ \pm}(\lambda) \in \mathbb{R}$ be the two solutions of $x_{k}^{0}\left(s_{0, k}^{ \pm}(\lambda)\right)=\lambda, \pm s_{0, k}^{ \pm}(\lambda)>0$, where $x_{k}^{0}$ is the unique solution of

$$
x^{\prime}=x\left(|y|^{p^{*}-2} y+p\right), \quad y^{\prime}=-x
$$

such that $x_{k}^{0}(0)=\bar{\lambda}^{k}$ and $y_{k}^{0}(0)=0$. With these notations, $\left(x_{1}^{0}, y_{1}^{0}\right)=\left(\bar{x}^{0}, \bar{y}^{0}\right)$ but $\left(x_{k}^{0}, y_{k}^{0}\right) \neq\left(\bar{x}^{0}, \bar{y}^{0}\right)$ for any $k \geq 2$. A careful rewriting of the Emden-Fowler change of variables then gives the following result.

Lemma 9 Let $\lambda \in\left(0, \bar{\lambda}_{k}^{+}\right]$for some $k \geq 1$. Then there exists two solutions $u^{ \pm}$of (12) which take the form

$$
e^{u^{ \pm}(r)}=\left[\frac{1}{\lambda r^{p}} \sum_{j=1}^{k} x_{j}^{0}\left(\log r+s_{k}(\varepsilon)+s_{0, k}^{ \pm}(\lambda)\right)\right](1+o(1)) \quad \forall r \in(0,1)
$$

as $\varepsilon \rightarrow 0$.

This actually amounts to say that there is a $k$-bubbles solution. Note that we have to assume that $\varepsilon>0$ is small enough so that with the notations of Corollary 8 ,

$$
a(\varepsilon)>s_{k}(\varepsilon)+s_{0, k}^{ \pm}(\lambda) .
$$

Theorem 10 Let $\varepsilon=n-p>0$ and consider any integer $k \geq 1$. For $\varepsilon>0$ small enough, consider a sequence $\left(\varepsilon_{i}\right)_{i \in \mathbb{N}}$ such that $0<\varepsilon_{i}<\varepsilon$ for any $i \in \mathbb{N}$ and $\lim _{i \rightarrow+\infty} \varepsilon_{i}=0$. Then, up to the extraction of a subsequence, there exist $k$ functions $v_{j}, j=1,2, \ldots k$, a sequence of $k$ parameters $\mu_{j}\left(\varepsilon_{i}\right), i \in \mathbb{N}, j=1,2, \ldots k$ for which

$$
\lim _{i \rightarrow+\infty}\left(\mu_{j+1}\left(\varepsilon_{i}\right)-\mu_{j}\left(\varepsilon_{i}\right)\right)=+\infty \quad \forall j=1,2, \ldots k-1
$$

such that any solution $u^{\varepsilon_{i}}$ of (5) with $\varepsilon=\varepsilon_{i}$ has the following property:

$$
e^{u^{\varepsilon_{i}}(x)}=\left[\frac{1}{|x|^{p}} \sum_{j=1}^{k} v_{j}\left(\log |x|+\mu_{j}\left(\varepsilon_{i}\right)\right)\right](1+o(1)) \quad \text { as } i \rightarrow+\infty
$$

uniformly on $B$.

\section{Lyapunov-Schmidt reduction: the Brezis-Nirenberg problem}

For $p=p^{*}+\varepsilon, p^{*}=\frac{n+2}{n-2}$, and a certain range $\lambda=o(1)$, depending on $\varepsilon$, one can see bubbling positive solutions by variational methods, or, to be precise, by a LyapunovSchmidt reduction. The method goes back to [20] but has been implemented in the 
framework of the Brezis-Nirenberg problem only recently [12]. There are actually towers of bubbles constituted by a superposition of bubbles of different blow-up orders: given any number $k \geq 1$, there is an $\varepsilon$-dependent range for $\lambda$ for which there exist solutions of the form

$$
u_{\varepsilon}(x)=\gamma_{n} \sum_{j=1}^{k} M_{j}\left(1+M_{j}^{4 /(n-2)}|x|^{2}\right)^{-(n-2) / 2}(1+o(1)) \quad \text { as } x \rightarrow 0,
$$

where $M_{j} \rightarrow+\infty$ and $M_{j}=o\left(M_{j+1}\right)$ for all $j$. This is in strong contrast with the case in which $\varepsilon=0$ and one lets $\lambda \downarrow 0$, or $\lambda=0$ and $\varepsilon \uparrow 0$, where only a single bubble is present, as established by Brezis and Peletier [6] (also see [29, 24]). For simplicity in the exposition, we restrict ourselves in this section to the case $n \geq 5$. The following result has been established in [12].

Theorem 11 [12] Assume $n \geq 5$ and $p=p^{*}+\varepsilon, p^{*}=\frac{n+2}{n-2}$. Then, given an integer $k \geq 1$, there exists a number $\mu_{k}>0$ such that if $\mu>\mu_{k}$ and

$$
\lambda=\mu \varepsilon^{\frac{n-4}{n-2}}
$$

then there are constants $0<\alpha_{j}^{-}<\alpha_{j}^{+}, j=1, \ldots k$ which depend on $k, n$ and $\mu$ and two solutions $u_{\varepsilon}^{ \pm}$of Problem (1) of the form (2) with $M_{j}=\alpha_{j}^{ \pm} \varepsilon^{\frac{1}{2}-j}$. In (2), o(1) $\rightarrow 0$ means that the convergence holds uniformly on $B$ as $\varepsilon \rightarrow 0$.

Proof. For completeness we shall sketch the proof of Theorem 11 along the lines of [12]. The problem of finding radial solutions $u$ to Problem (1) corresponds to that of solving the boundary value problem

$$
u^{\prime \prime}+\frac{n-1}{r} u^{\prime}+u^{p+\varepsilon}+\lambda u=0, \quad u^{\prime}(0)=0, \quad u(1)=0 .
$$

Here and in the rest of this section we fix $p^{*}=\frac{n+2}{n-2}$ and we write simply $u=u(r)$ with $r=|x|$. We transform the problem by means of the following change of variable

$$
v(s)=\left(\frac{2}{p^{*}-1}\right)^{-2 /(p-1)} r^{2 /\left(p^{*}-1\right)} u(r) \quad \text { with } r=e^{-\left(p^{*}-1\right) s / 2}, \quad s \in(0,+\infty),
$$

a variation of the so-called Emden-Fowler transformation, first introduced in [21]. Note that this is not exactly the change of variables introduced in Section 2.1. Problem (14) then becomes

$$
\left\{\begin{array}{l}
v^{\prime \prime}-v+e^{\varepsilon s} v^{p}+\left(\frac{p^{*}-1}{2}\right)^{2} \lambda e^{-\left(p^{*}-1\right) s} v=0 \quad \text { on }(0, \infty), \\
v(0)=0, \quad v>0, \quad v(s) \rightarrow 0 \text { as } s \rightarrow+\infty
\end{array}\right.
$$

The energy functional associated to Problem (16) is given by

$$
E_{\varepsilon}(v)=I_{\varepsilon}(v)-\frac{1}{8}\left(p^{*}-1\right)^{2} \lambda \int_{0}^{\infty} e^{-\left(p^{*}-1\right) s}|v|^{2} d s
$$

with

$$
I_{\varepsilon}(v)=\frac{1}{2} \int_{0}^{\infty}\left|v^{\prime}\right|^{2} d s+\frac{1}{2} \int_{0}^{\infty}|v|^{2} d s-\frac{1}{p+1} \int_{0}^{\infty} e^{\varepsilon s}|v|^{p+1} d s .
$$

Let us consider the unique positive solution $\mathcal{V}$ to the problem

$$
\left\{\begin{array}{l}
\mathcal{V}^{\prime \prime}-\mathcal{V}+\mathcal{V}^{p^{*}}=0 \text { on }(-\infty, \infty) \\
\mathcal{V}^{\prime}(0)=0 \\
\mathcal{V}>0, \quad \mathcal{V}(s) \rightarrow 0 \text { as } s \rightarrow \pm \infty
\end{array}\right.
$$


This solution is nothing but the one given by the Emden-Fowler transformation (15) of the radial positive solution of $\Delta v+v^{p^{*}}=0$ given by

$$
\mathcal{V}(s)=\left(\frac{4 n}{n-2}\right)^{\frac{n-2}{4}} e^{-s}\left(1+e^{-\frac{4}{n-2} s}\right)^{-\frac{n-2}{2}} .
$$

Let us consider points $0<\xi_{1}<\xi_{2}<\cdots<\xi_{k}$. We look for a solution of (16) of the form

$$
v(s)=\sum_{i=1}^{k}\left(\mathcal{V}\left(s-\xi_{i}\right)+\pi_{i}\right)+\phi
$$

where $\phi$ is small and $\pi_{i}(s)=-\mathcal{V}\left(\xi_{i}\right) e^{-s}$. The correction $\pi_{i}$ is meant to make the ansatz satisfy the Dirichlet boundary conditions. A main observation is that $v(s) \sim$ $\sum_{i=1}^{k} \mathcal{V}\left(s-\xi_{i}\right)$ solves (16) if and only if going back in the change of variables, with $\gamma_{n}=s(n(n-2))^{(n-2) / 4}$,

$$
u(r) \sim \gamma_{n} \sum_{i=1}^{k}\left(1+e^{\frac{4 \xi_{i}}{n-2}} r^{2}\right)^{-(n-2) / 2} e^{\xi_{i}}
$$

solves (14). Therefore the ansatz given for $v$ provides (for large values of the $\xi_{i}$ 's), a bubble-tower solution for (1) of the form (2) with $M_{i}=e^{\xi_{i}}$.

Let us write

$$
\mathcal{V}_{i}(s)=\mathcal{V}\left(s-\xi_{i}\right), \quad V_{i}=\mathcal{V}_{i}+\pi_{i}, \quad \pi_{i}(s)=-\mathcal{V}\left(\xi_{i}\right) e^{-s}, \quad V=\sum_{i=1}^{k} V_{i}
$$

It is easily checked that $V_{i}$ is nonnegative on $\mathbb{R}^{+}$. We shall work out asymptotics for the associated energy functional evaluated at the function $V$, assuming that the numbers $\xi_{i}$ take the form

$$
\begin{gathered}
\xi_{1}=-\frac{1}{2} \log \varepsilon+\log \Lambda_{1}, \\
\xi_{i+1}-\xi_{i}=-\log \varepsilon-\log \Lambda_{i+1}, \quad i=1, \ldots k-1,
\end{gathered}
$$

where the $\Lambda_{i}$ 's are positive parameters. For notational convenience, we also set $\Lambda=$ $\left(\Lambda_{1}, \Lambda_{2}, \ldots \Lambda_{k}\right)$. With this choice the energy $E_{\varepsilon}$ defined by (17) can be expanded as follows.

Lemma 12 Let $n \geq 5$. Fix a small number $\delta>0$ and assume that

$$
\delta<\Lambda_{i}<\delta^{-1} \quad \text { for all } i=1, \ldots k .
$$

Assume also that $\lambda=\mu \varepsilon^{\frac{n-4}{n-2}}$ for some $\mu>0$. Let $V$ be given by (18). Then, with the choice (19) of the points $\xi_{i}$, there are positive numbers $a_{i}, i=0, \ldots 5$ depending only on $n$, such that the following expansion holds:

$$
\begin{aligned}
& E_{\varepsilon}(V)=k a_{0}+\varepsilon \Psi_{k}(\Lambda)+\frac{k^{2}}{2} a_{3} \varepsilon \log \varepsilon+a_{5} \varepsilon+\varepsilon \theta_{\varepsilon}(\Lambda), \text { where } \\
& \Psi_{k}(\Lambda)=a_{1} \Lambda_{1}^{-2}-k a_{3} \log \Lambda_{1}-a_{4} \mu \Lambda_{1}^{-\left(p^{*}-1\right)}+\sum_{i=2}^{k}\left[(k-i+1) a_{3} \log \Lambda_{i}-a_{2} \Lambda_{i}\right]
\end{aligned}
$$

and as $\varepsilon \rightarrow 0$, the term $\theta_{\varepsilon}(\Lambda)$ converges to 0 uniformly and in the $C^{1}$-sense on the set of $\Lambda_{i}$ 's satisfying contraints (20). 
Indeed, if there is a solution of (16) of the form $v=V+\phi$, with $V$ as in the statement of the lemma, and $\phi$ small, it is natural to expect that this occurs if the vector $\Lambda=\left(\Lambda_{1}, \ldots \Lambda_{k}\right)$ corresponds to a critical point of the function $\Psi_{k}$. This is in fact true, as it follows from a Lyapunov-Schmidt reduction procedure. Before, let us analyze the critical points of $\Psi_{k}$ :

$$
\begin{gathered}
\Psi_{k}(\Lambda)=\varphi_{k}^{\mu}\left(\Lambda_{1}\right)+\sum_{i=2}^{k} \varphi_{i}\left(\Lambda_{i}\right), \\
\varphi_{k}^{\mu}(s)=a_{1} s^{-2}-k a_{3} \log s-a_{4} \mu s^{-\left(p^{*}-1\right)} \text { and } \varphi_{i}(s)=(k-i+1) a_{3} \log s-a_{2} s .
\end{gathered}
$$

Let us observe that there is a number $\mu_{k}>0$ such that $\varphi_{k}^{\mu}$ has exactly two critical points: a nondegenerate maximum, $s_{k}^{+}(\mu)$, and a nondegenerate minimum, $s_{k}^{-}(\mu)$. On the other hand, each of the functions $\varphi_{j}$ has exactly one nondegenerate critical point, a maximum,

$$
s=(k-j+1) b_{3}, \quad \text { for each } j=2, \ldots k,
$$

with $b_{3}$ is a given positive constant depending on $n$.

Lemma 13 Assume that $\mu>\mu_{k}$. Then, the function $\Psi_{k}(\Lambda)$ has exactly two critical points, given by

$$
\Lambda^{ \pm}=\left(s_{k}^{ \pm}(\mu),(k-1) b_{3},(k-2) b_{3}, \ldots b_{3}\right) .
$$

These critical points are nondegenerate.

Let us consider again points $0<\xi_{1}<\xi_{2}<\ldots \xi_{k}$, which are for now arbitrary. We keep the notations $\mathcal{V}_{i}, V_{i}$ and $V$ defined by (18). Additionally we define

$$
Z_{i}(s)=\mathcal{V}_{i}^{\prime}(s)-\mathcal{V}_{i}^{\prime}(0) e^{-s}, \quad i=1, \ldots k
$$

and consider the problem of finding a function $\phi$ for which there are constants $c_{i}$, $i=1, \ldots k$, such that, in $(0, \infty)$

$$
\left\{\begin{array}{l}
-(V+\phi)^{\prime \prime}+(V+\phi)-e^{\varepsilon s}(V+\phi)_{+}^{p}-\lambda\left(\frac{p^{*}-1}{2}\right)^{2} e^{-\left(p^{*}-1\right) s}(V+\phi)=\sum_{i=1}^{k} c_{i} Z_{i}, \\
\phi(0)=0, \quad \lim _{s \rightarrow+\infty} \phi(s)=0, \\
\int_{0}^{\infty} Z_{i} \phi d s=0 \quad \text { for all } i=1, \ldots k .
\end{array}\right.
$$

This problem turns out to be solvable for points $\xi_{i}$ chosen in a convenient range. After this, the original problem becomes reduced to adjusting the points $\xi_{i}$ so that $c_{i}=0$ for all $i$.

In order to solve Problem (22), let us consider the linearized operator around $V$ defined as

$$
\mathcal{L}_{\varepsilon} \phi=-\phi^{\prime \prime}+\phi-p e^{\varepsilon s} V^{p-1} \phi-\lambda\left(\frac{p^{*}-1}{2}\right)^{2} e^{-\left(p^{*}-1\right) s} \phi .
$$

Then Problem (22) can be rewritten as

$$
\left\{\begin{array}{l}
\mathcal{L}_{\varepsilon} \phi=N_{\varepsilon}(\phi)+R_{\varepsilon}+\sum_{i=1}^{k} c_{i} Z_{i} \quad \text { in }(0, \infty) \\
\phi(0)=0, \quad \lim _{s \rightarrow+\infty} \phi(s)=0 \\
\int_{0}^{\infty} Z_{i} \phi d s=0 \quad \text { for all } i=1, \ldots k
\end{array}\right.
$$


where

$$
\begin{aligned}
& N_{\varepsilon}(\phi)=e^{\varepsilon s}\left[(V+\phi)_{+}^{p}-V^{p}-p V^{p-1} \phi\right] \text { and } \\
& R_{\varepsilon}=e^{\varepsilon s}\left[V^{p}-V^{p^{*}}\right]+V^{p^{*}}\left[e^{\varepsilon s}-1\right]+\left[V^{p^{*}}-\sum_{i=1}^{k} V_{i}^{p^{*}}\right]+\lambda\left(\frac{p^{*}-1}{2}\right)^{2} e^{-\left(p^{*}-1\right) s} V .
\end{aligned}
$$

The operator $\mathcal{L}_{\varepsilon}$ turns out to be boundedly invertible under the orthogonality conditions, for an appropriate norm which depends on the points $\xi_{i}$. For a small, fixed positive number $\sigma$ and a function $\psi$ defined on $(0, \infty)$, let us set

$$
\|\psi\|_{*}=\sup _{s>0}\left(\sum_{i=1}^{k} e^{-\sigma\left|s-\xi_{i}\right|}\right)^{-1}|\psi(s)| .
$$

Consider the linear problem of, given a function $h$, finding $\phi$ such that

$$
\left\{\begin{array}{l}
\mathcal{L}_{\varepsilon} \phi=h(s)+\sum_{i=1}^{k} c_{i} Z_{i} \text { in }(0, \infty), \\
\phi(0)=0, \quad \lim _{s \rightarrow+\infty} \phi(s)=0, \\
\int_{0}^{\infty} Z_{i} \phi d s=0 \text { for all } i=1, \ldots k,
\end{array}\right.
$$

for certain constants $c_{i}$.

Lemma 14 There exist positive numbers $\varepsilon_{0}, \delta_{0}, \delta_{1}, R_{0}$, and a constant $C>0$ such that if the scalar $\lambda$ and the points $0<\xi_{1}<\xi_{2}<\cdots<\xi_{k}$ satisfy

$$
R_{0}<\xi_{1}, \quad R_{0}<\min _{1 \leq i<k}\left(\xi_{i+1}-\xi_{i}\right), \quad \xi_{k}<\frac{\delta_{0}}{\varepsilon}, \quad \lambda<\delta_{1},
$$

then for all $0<\varepsilon<\varepsilon_{0}$ and all $h \in C[0, \infty)$ with $\|h\|_{*}<+\infty$, Problem (26) admits a unique solution $\phi=: T_{\varepsilon}(h)$. Besides,

$$
\left\|T_{\varepsilon}(h)\right\|_{*} \leq C\|h\|_{*} \quad \text { and } \quad\left|c_{i}\right| \leq C\|h\|_{*} .
$$

Now we are ready to solve Problem (22). We shall do this after restricting conveniently the range of the parameters $\xi_{i}$ and $\lambda$. Let us consider for a number $M$ large but fixed, the following conditions:

$$
\left\{\begin{array}{c}
\xi_{1}>\frac{1}{2} \log (M \varepsilon)^{-1}, \quad \log (M \varepsilon)^{-1}<\min _{1 \leq i<k}\left(\xi_{i+1}-\xi_{i}\right), \\
\xi_{k}<k \log (M \varepsilon)^{-1}, \quad \lambda<M \varepsilon^{\frac{n-4}{n-2}}
\end{array}\right.
$$

Useful facts that we easily check is that under relations (28), $N_{\varepsilon}$ and $R_{\varepsilon}$ defined by (24) satisfy for all small $\varepsilon>0$ and $\|\phi\|_{*} \leq \frac{1}{4}$ the estimates:

$$
\left\|N_{\varepsilon}(\phi)\right\|_{*} \leq C\|\phi\|_{*}^{p^{*}} \text { and }\left\|R^{\varepsilon}\right\|_{*} \leq C \varepsilon^{1-\sigma}
$$

provided that $\sigma$ is chosen small enough.

Lemma 15 Assume that relations (28) hold. Then there is a constant $C>0$ such that, for all $\varepsilon>0$ small enough, there exists a unique solution $\phi=\phi(\xi)$ to problem (22) which besides satisfies

$$
\|\phi\|_{*} \leq C \varepsilon^{1-\sigma} .
$$

Moreover, the map $\xi \mapsto \phi(\xi)$ is of class $C^{1}$ for the $\|\cdot\|_{*}$-norm and

$$
\left\|D_{\xi} \phi\right\|_{*} \leq C \varepsilon^{1-\sigma} .
$$


Proof. We will only prove that the existence statement of (22) is equivalent to solving a fixed point problem. Indeed $\phi$ is a solution of (22) if and only if

$$
\phi=T_{\varepsilon}\left(N_{\varepsilon}(\phi)+R_{\varepsilon}\right)=: A_{\varepsilon}(\phi) .
$$

Thus it is sufficient to prove that the operator $A_{\varepsilon}$ defined above is a contraction in a proper region. Let us consider the set

$$
\mathcal{F}_{r}=\left\{\phi \in C[0, \infty):\|\phi\|_{*} \leq r \varepsilon^{1-\sigma}\right\}
$$

with $r$ a positive number to be fixed later. From Proposition 14 and (29), we get

$$
\left\|A_{\varepsilon}(\phi)\right\|_{*} \leq C\left\|N_{\varepsilon}(\phi)+R_{\varepsilon}\right\|_{*} \leq C\left[(r \varepsilon)^{p^{*}}+\varepsilon^{1-\sigma}\right]<r \varepsilon^{1-\sigma}
$$

for all small $\varepsilon$, provided that $r$ is chosen large enough, but independent of $\varepsilon$. Thus $A_{\varepsilon}$ maps $\mathcal{F}_{r}$ into itself for this choice of $r$. Moreover, $A_{\varepsilon}$ turns out to be a contraction mapping in this region. This follows from the fact that $N_{\varepsilon}$ defines a contraction in the

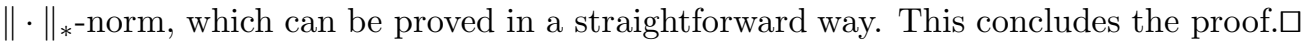

Now let us fix a large number $M$ and assume that conditions (28) hold true for $\xi=\left(\xi_{1}, \ldots \xi_{k}\right)$ and $\lambda$. According to the previous results, our problem has been reduced to that of finding points $\xi_{i}$ so that the constants $c_{i}$ which appear in (23), for the solution $\phi$ given by Lemma 15, are all zero. Thus we need to solve the system of equations

$$
c_{i}(\xi)=0 \text { for all } i=1, \ldots k .
$$

If (30) holds, then $v=V+\phi$ will be a solution to (22) with the desired form. This system turns out to be equivalent to a variational problem, which we introduce next.

Let us consider the functional

$$
\mathcal{I}_{\varepsilon}(\xi)=E_{\varepsilon}(V+\phi),
$$

where $\phi=\phi(\xi)$ is given by Lemma 15 and $E_{\varepsilon}$ is defined by (17). We claim that solving system (30) is equivalent to finding a critical point of this functional. In fact, integrating (22) against $Z_{i}$ and using the definition of $E_{\varepsilon}$ and $\phi$, we obtain

$$
D E_{\varepsilon}(V+\phi)\left[Z_{i}\right]=0 \text { for all } i=1, \ldots k .
$$

Now, it is easily checked that

$$
\frac{\partial}{\partial \xi_{i}}(V+\phi)=Z_{i}+o(1)
$$

with $o(1) \rightarrow 0$ in the ${ }^{*}$-norm as $\varepsilon \rightarrow 0$. We can decompose each of the $o(1)$ terms above as the sum of a small term which lies in the vector space spanned by the $Z_{i}$ 's, and a function $\eta$ with $\int_{0}^{+\infty} Z_{i} \eta d s=0$ for all $i$. Again, from equation (22), we get $D J_{\varepsilon}(V+\phi)[\eta]=0$. What we have shown is that system (31) is equivalent to

$$
\nabla \mathcal{I}_{\varepsilon}(\xi)=0 .
$$

The following fact is crucial to find critical points of $\mathcal{I}_{\varepsilon}$.

Lemma 16 Assume that $\sigma<\frac{1}{2}$ in the definition of the $*$-norm. Then the following expansion holds

$$
\mathcal{I}_{\varepsilon}(\xi)=E_{\varepsilon}(V)+o(\varepsilon),
$$

where the term $o(\varepsilon)$ is uniform in the $C^{1}$-sense over all points satisfying constraint (28), for given $M>0$. 
Proof of Theorem 11. Let us assume $\mu>\mu_{k}$. We need to find a critical point of $\mathcal{I}_{\varepsilon}(\xi)$. Consider the change of variable $\xi=\xi(\Lambda)$

$$
\xi_{1}=-\frac{1}{2} \log \varepsilon-\log \Lambda_{1}, \quad \xi_{i+1}-\xi_{i}=-\log \varepsilon-\log \Lambda_{i}, \quad i \geq 2,
$$

where the $\Lambda_{i}$ 's are positive parameters, and define $\Lambda:=\left(\Lambda_{1}, \ldots \Lambda_{k}\right)$. Thus it suffices to find a critical point of

$$
\Phi_{\varepsilon}(\Lambda) \equiv \varepsilon^{-1} \nabla \mathcal{I}_{\varepsilon}(\xi(\Lambda)) .
$$

From the above lemma and the decomposition (21) given in Lemma 12, which actually holds with the $o(\varepsilon)$ term in the $C^{1}$ sense uniformly on points satisfying constraints (28), we obtain

$$
\nabla \Phi_{\varepsilon}(\Lambda)=\nabla \Psi_{k}(\Lambda)+o(1),
$$

where $o(1) \rightarrow 0$ uniformly on points $\Lambda$ satisfying (20). We assume that for our fixed $\mu>\mu_{k}$, the critical points $\Lambda^{ \pm}$of $\Psi_{k}$ in Lemma 16 satisfy this constraint. Since the critical points $\Lambda^{ \pm}$are nondegenerate, it follows that the local degrees $\operatorname{deg}\left(\nabla \Psi_{k}, \mathcal{N}_{ \pm}, 0\right)$ are well defined and they are non-zero. Here $\mathcal{N}_{ \pm}$are arbitrarily small neighborhoods of the points $\Lambda^{ \pm}$in $\mathbb{R}^{k}$. We also conclude that $\operatorname{deg}\left(\nabla \mathcal{I}_{\varepsilon}, \mathcal{N}_{ \pm}, 0\right) \neq 0$ for all sufficiently small $\varepsilon$. Hence we may find critical points $\Lambda_{\varepsilon}^{ \pm}$of $\Phi_{\varepsilon}$ with

$$
\Lambda_{\varepsilon}^{ \pm}=\Lambda^{ \pm}+o(1), \quad \lim _{\varepsilon \rightarrow 0} o(1)=0 .
$$

For $\xi_{\varepsilon}^{ \pm}=\xi\left(\Lambda_{\varepsilon}^{ \pm}\right)$, the functions $v^{ \pm}=V+\phi\left(\xi_{\varepsilon}^{ \pm}\right)$are solutions of Problem (16). From the equation satisfied by $\phi,(22)$, and its smallness in the $*$-norm, we derive that $v=V(1+o(1))$, where $o(1) \rightarrow 0$ uniformly on $(0, \infty)$. Further, if we simply set $\xi^{ \pm} \equiv \xi\left(\Lambda^{ \pm}\right)$, then it is also true that

$$
v^{ \pm}(s)=\sum_{1=1}^{k} \mathcal{V}\left(s-\xi_{i}^{ \pm}\right)(1+o(1)),
$$

again with $o(1) \rightarrow 0$ uniformly on $(0, \infty)$. Finally, if we go back in the change of variables (15) to a solution of (1), the explicit form of the parameters $\Lambda^{ \pm}$found in Lemma 13 provides the expression (2) for the solutions. This concludes the proof of Theorem 11.

\section{Conclusion}

The variational approach for the Brezis-Nirenberg problem raises plenty of questions when the domain is not a ball. For a review of recent results in this direction see [17].

The Brezis-Nirenberg problem and the equation corresponding to the case of the exponential nonlinearity share very similar properties in the slightly supercritical regime when they are studied by phase plane methods. We have shown that both problems have the multi-bubbling property, which means that in the phase space, the solutions make an arbitrarily large number of "turns". This property is reflected by the "oscillations" of the bifurcation diagram.

However, the results for the exponential nonlinearity are still quite preliminary. The uniqueness of the "heights" of the bubbles and of course their characterization is still unknown. This might be tackled by variational methods as for the Brezis-Nirenberg problem (and eventually extended to domains with more general geometries than a ball) but for the moment the problem is still completely open.

Acknowledgment Partially supported by ECOS-Conicyt under contract C02E08, by the CMM (UMR CNRS no. 2071), Universidad de Chile, by the EU financed network HPRN-CT-2002-00282 and by the Amadeus constracts no. 02580QF and 05538SB. 


\section{References}

[1] F.V. Atkinson, H. Brezis, L.A. Peletier, Nodal solutions of elliptic equations with critical Sobolev exponents, J. Differential Equations 85 no. 1 (1990), 151-170.

[2] F.V. Atkinson, L.A. Peletier, Large solutions of elliptic equations involving critical exponents, Asymptotic Anal. 1 (1988), 139-160.

[3] R.D. Benguria, J. Dolbeault, M.J. Esteban, Classification of the solutions of semilinear elliptic problems in a ball, J. Differential Equations 167 no. 2 (2000), 438-466.

[4] P. Biler, J. Dolbeault, M. J. Esteban, P. Markowich, T. Nadzieja, Steady states for Streater's energy-transport models of self-gravitating particles, to appear in IMA Vol. Math. Appl., Springer.

[5] H. Brezis, L. Nirenberg, Positive solutions of nonlinear elliptic equations involving critical Sobolev exponents, Comm. Pure Appl. Math. 36 no. 4 (1983), 437-477.

[6] H. Brezis, L.A. Peletier, Asymptotics for elliptic equations involving critical growth, Partial differential equations and the calculus of variations, Vol. I, 149-192, Progr. Nonlinear Differential Equations Appl. 1, Birkhauser Boston, 1989.

[7] F. Brock, Continuous rearrangement and symmetry of solutions of elliptic problems, Proc. Indian Acad. Sci. Math. Sci. 110 no. 2 (2000), 157-204.

[8] C. Budd, J. Norbury, Semilinear elliptic equations and supercritical growth, J. Differential Equations 68 no. 2 (1987), 169-197.

[9] X. CABRÉ, Extremal solutions and instantaneous complete blow-up for elliptic and parabolic equations, Preprint.

[10] X. Cabré, Y. MARTEL, Existence versus explosion instantanée pour des équations de la chaleur linéaires avec potentiel singulier (Existence versus instantaneous blow-up for linear heat equations with singular potentials), C. R. Acad. Sci., Paris, Sér. I, Math. 329 no. 11 (1999), 973-978.

[11] L. Damascelli, F. Pacella, M. Ramaswamy, Symmetry of Ground States of p-Laplace Equations via the Moving Plane Method, Arch. Rational Mech. Anal. 148 no. 4 (1999), 291-308.

[12] M. Del Pino, J. Dolbeault, M. Musso, "Bubble-tower" radial solutions in the slightly supercritical Brezis-Nirenberg problem, Preprint Ceremade no. 0214, to appear in J. Differential Eq.

[13] M. Del Pino, J. Dolbeault, M. Musso, Multi-bubbling in some slightly supercritical equations: the case of the exponential nonlinearity, in preparation.

[14] M. Del Pino, J. Dolbeault, M. Musso, Duality in sub-and super-critical bubbling in the Brezis-Nirenberg problem, in preparation.

[15] M. Del Pino, P. Felmer, M. Musso, Two-bubble solutions in the super-critical Bahri-Coron problem, Calc. Var. Partial Differential Equations 16 no. 2 (2003), 113-145.

[16] M. Del Pino, P. Felmer, M. Musso, Multi-peak solution for super-critical elliptic problems in domains with small holes, J. Differential Equations 182 no. 2 (2002), 511-540.

[17] M. Del Pino, M. Musso, Super-critical bubbling in elliptic boundary value problems, to appear.

[18] J. Dolbeault, M.J. Esteban, M. Ramaswamy, Radial Singular Solutions of a Critical Problem in a Ball, Differential Integral Equations 15 no. 12 (2002), 1459-1474. 
[19] O. Druet, Elliptic equations with critical Sobolev exponent in dimension 3, Ann. Inst. H. Poincaré Anal. Non Linéaire 19 no. 2 (2002), 125-142.

[20] A. Floer, A. Weinstein, Nonspreading wave packets for the cubic Schrödinger equation with a bounded potential, J. Funct. Anal. 69 no. 3 (1986), 397-408.

[21] R.H. Fowler, Further studies on Emden's and similar differential equations, Quart. J. Math. 2 (1931), 259-288.

[22] I. M. Gelfand, Some problems in the theory of quasi-linear equations, Amer. Math. Soc. Transl. 29 (1963), 295-381.

[23] B. Gidas, W.M. Ni, L. Nirenberg, Symmetry and related properties via the Maximum Principle, Commun. Math. Phys. 68 (1979), 209-243.

[24] Z.-C. HAN, Asymptotic approach to singular solutions for nonlinear elliptic equations involving critical Sobolev exponent, Ann. Inst. H. Poincaré Anal. Non Linéaire 8 no. 2 (1991), 159-174.

[25] D. D. Joseph, T. S. Lundgren, Quasilinear problems driven by positive sources, Arch. Rat. Mech. Anal. 49 (1973), 241-269.

[26] M.K. Kwong, Y. Li, Uniqueness of radial solutions of semilinear elliptic equations, Trans. A.M.S. 333 (1992), 339-363.

[27] F. Merle, L.A. Peletier, Positive solutions of elliptic equations involving supercritical growth, Proc. Roy. Soc. Edinburgh Sect. A 118 no. 1-2 (1991), 49-62.

[28] F. Mignot, J.-P. Puel, Quelques résultats sur un problème elliptique avec non linéarité exponentielle [Some results on an elliptic problem with exponential nonlinearity], Équations aux dérivées partielles et applications, 683-704, GauthierVillars, Éd. Sci. Méd. Elsevier, Paris, 1998.

[29] O. REY, The role of the Green's function in a nonlinear elliptic equation involving the critical Sobolev exponent, J. Funct. Anal. 89 no. 1 (1990), 1-52.

[30] J. Serrin, H. Zou, Classification of positive solutions of quasilinear elliptic equations, Topol. Methods Nonlinear Anal. 3 no. 1 (1994), 1-25.

[31] L.Q. ZHANG, Uniqueness of positive solutions to semilinear elliptic equations, Acta Math. Sci. (Chinese) 11 no. 2 (1991), 130-142.

[32] L.Q. ZhANG, Uniqueness of positive solutions of $\Delta u+u+u^{p}=0$ in a finite ball, Comm. P.D.E. 17 (1992), 1141-1164. 


\section{Figures}

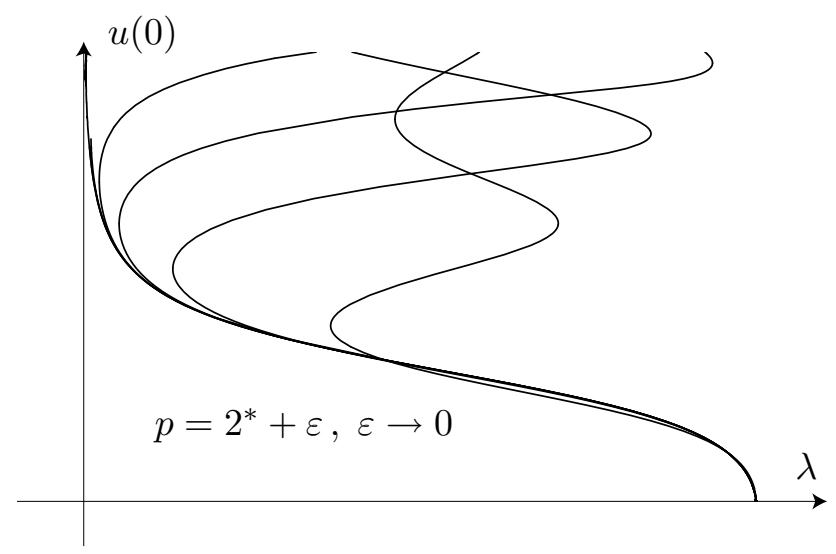

Figure 1: Bifurcation diagrams of the Brezis-Nirenberg problem (1) in the critical and slightly supercritical cases.

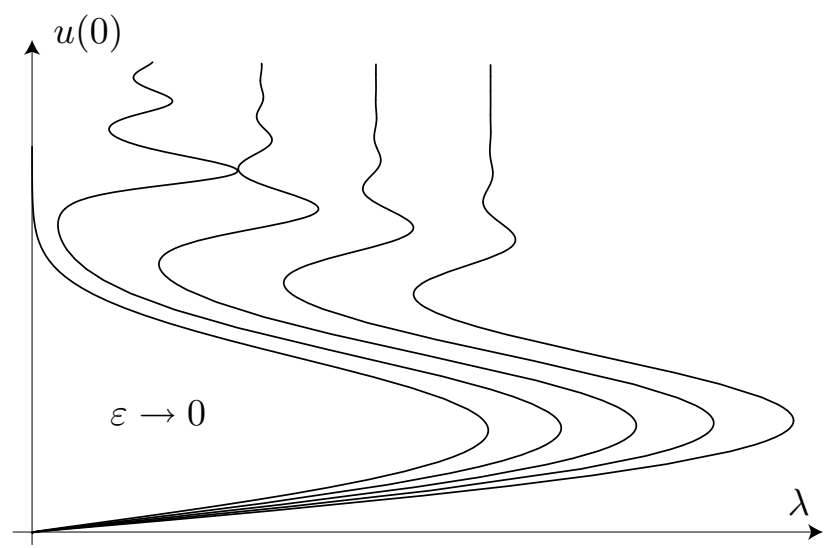

Figure 2: Bifurcation diagrams of the problem with the exponential nonlinearity (5) in the critical and slightly supercritical cases $(n=2, p=2-\varepsilon)$. The fact that the nonlinearity $u \mapsto e^{u}$ takes a non zero value at $u=0$ is responsible for the fact that the branch of minimal solutions bifurcates from $(0,0)$. This is of course a fundamental difference with the Brezis-Nirenberg problem (1) which bifurcates from $\left(\lambda_{1}, 0\right)$. 


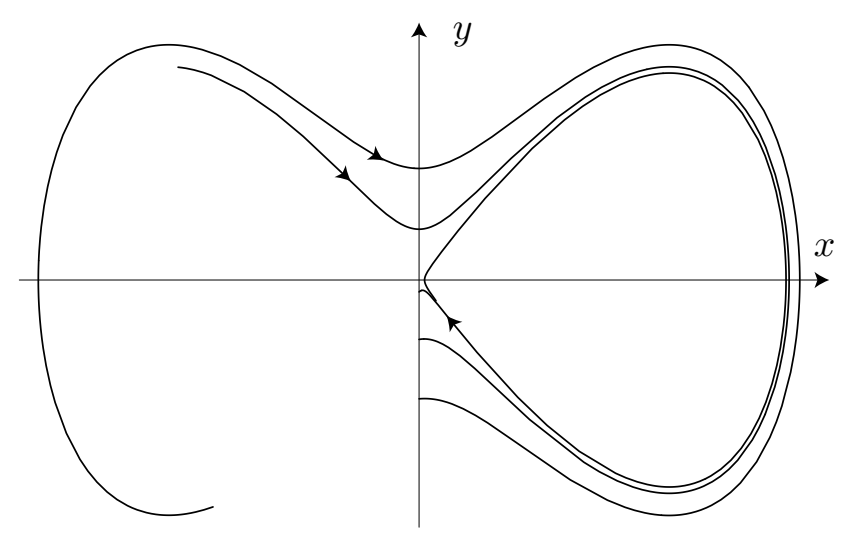

Figure 3: Phase portrait for the Brezis-Nirenberg problem after the Emden-Fowler transformation of Section 2.1 in the slightly supercritical case.

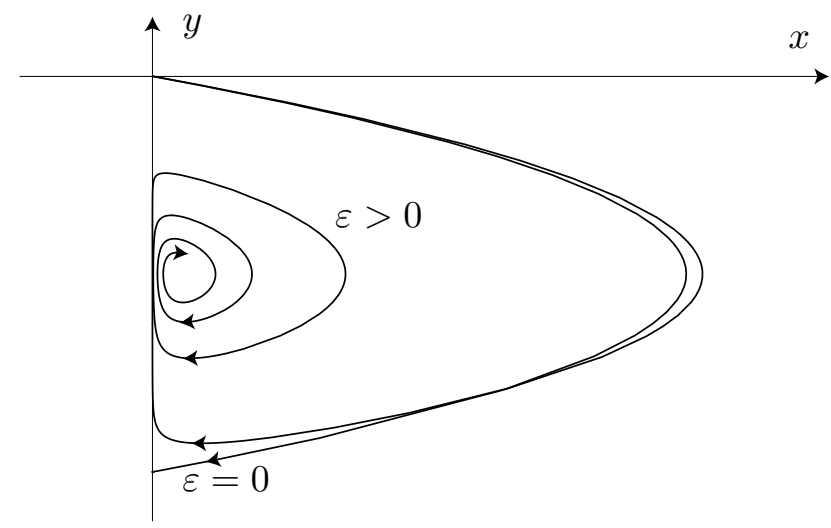

Figure 4: Phase portrait for the problem with the exponential nonlinearity after the Emden-Fowler transformation in the slightly supercritical case. 